S. GROEGER ${ }^{1,2,}$ A.S. PAZGALEV ${ }^{1,3}$ A. WEIS ${ }^{1}$

\title{
Comparison of discharge lamp and laser pumped cesium magnetometers
}

\author{
${ }^{1}$ Physics Department, Université de Fribourg, Chemin de Musée 3, 1700 Fribourg, Switzerland \\ ${ }^{2}$ Paul Scherrer Institute, 5232 Villigen PSI, Switzerland \\ ${ }^{3}$ Ioffe Physical Technical Institute, Russian Academy of Sciences, St. Petersburg 194021, Russia
}

\section{Received: 30 November 2004 / \\ Revised version: 20 January 2005 / \\ Published online: 17 March 2005 • (c) Springer-Verlag 2005}

ABSTRACT We have performed a comparison of laser (LsOPM) and lamp (LpOPM) pumped cesium vapor magnetometers. Although the LsOPM operated 50\% above its shot-noise limit we found an intrinsic sensitivity of $15 \mathrm{fT} / \sqrt{\mathrm{Hz}}$ and $25 \mathrm{fT} / \sqrt{\mathrm{Hz}}$ for the LsOPM and the LpOPM, respectively. Two modes of operation, viz. the phase-stabilized and the self-oscillating modes, were investigated and found to yield a similar performance. We have compared the performance of the LsOPM and the LpOPM directly by simultaneous measurements of field fluctuations of a $2-\mu \mathrm{T}$ magnetic field inside a multilayer magnetic shield and have used one of the magnetometers for an active field stabilization. In the stabilized mode we found a gradient instability of $25 \mathrm{fT}$ within an integration time of $100 \mathrm{~s}$, which represents an upper limit of the long-term stability of the magnetometers. Our research is motivated by the need for an improved control of magnetic fields and gradients in a planned neutron electric dipole experiment.

PACS 07.55.Ge; 32.30.Dx

1

\section{Introduction}

The precise measurement and control of magnetic fields and field fluctuations is of crucial importance in many fundamental physics experiments. The suppression of systematic uncertainties in experiments searching for permanent electric dipole moments (EDMs) in atoms and neutrons is one prominent example. New generations of EDM experiments with ultra-cold neutrons (UCNs) aim at putting more stringent limits on (or even observing) an EDM by the use of higher UCN flux and larger storage volumes, which improve the statistical sensitivity. The increased sensitivity also puts more stringent constraints on systematic effects and calls, in particular, for a better control of the stability of the magnetic field and its gradients. Although magnetometers based on superconducting quantum interference devices (SQUIDs) are the most sensitive magnetometers available to date, they are of limited interest for monitoring magnetic fields in large

Fax: +41-26-3009631,E-mail: stephan.groeger@unifr.ch volumes. Moreover, SQUIDs do not measure absolute field values.

Two distinct magnetometric techniques were used in past EDM experiments. In the ILL experiment [1], which has produced the presently lowest upper bound on the neutron EDM, a vapor of ${ }^{199} \mathrm{Hg}$ atoms filled into the ultra-cold neutron storage chamber (201) served as a 'cohabitating' magnetometer. The PNPI experiment [2], on the other hand, used a set of two selfoscillating cesium vapor magnetometers placed above and below the storage chamber for monitoring the field in the chamber. Both techniques have pros and cons. Co-magnetometers yield only a volume-averaged field value, which yields no information on field gradients and their fluctuations. External magnetometers, on the other hand, do not measure the field in the volume of interest directly, but allow us - if used in sufficient number - to access field distributions, thereby permitting the active control of specific multipole moments of the field. Borisov et al. have proposed a large-volume (external) magnetometer based on nuclear spin precession in ${ }^{3} \mathrm{He}$ [3]. That device uses a double pulse Ramsey resonance technique, which besides its lack of spatial resolution also suffers from a lack of temporal resolution.

The PNPI experiment used two conventional state-of-theart discharge lamp pumped self-oscillating cesium vapor magnetometers (OPMs) [2]. Such types of magnetometers - developed since the 1950s - have a shot-noise-limited performance and large bandwidths. The high spatial and temporal resolution of optically pumped alkali magnetometers thus make such devices interesting alternatives for the continuous monitoring of fields, gradients, and fluctuations thereof. The use of alkali OPMs for the field control in larger volumes calls for a substantially larger number of sensor heads, which suffers from the fact that a single discharge lamp can only drive a limited number of sensors. The steady development in the past decades of narrow-band semiconductor diode lasers makes such light sources attractive alternatives to discharge lamps. Owing to the high spectral density of its radiation, a single diode laser of moderate power (a few $\mathrm{mW}$ ) can be used to drive dozens of magnetometer heads.

Having a multichannel external magnetometer approach for a planned neutron EDM experiment in mind, we have performed a comparative study of state-of-the-art discharge lamp pumped magnetometers (LpOPM) and laser pumped 
magnetometers (LsOPM) using similar room-temperature sensor cells (7- and 6-cm diameter, respectively) and identical electronics. We discuss the principle of operation and details of their practical realization. The devices were operated in two distinct modes, viz. the self-oscillating mode and the phase-stabilized mode. Details of the development and performance of the LsOPM will be published elsewhere [4]. We have determined the intrinsic sensitivities of the magnetometers and present measurements of the fluctuations of a $2-\mu \mathrm{T}$ field recorded simultaneously by the LsOPM and the LpOPM in a multilayer magnetic shield. The LpOPM reached its ultimate shot-noise-limited performance while the LsOPM showed a superior intrinsic sensitivity, although its performance still lies 50\% above its fundamental shot-noise limit.

\section{$2 \quad$ Optically pumped magnetometers}

\section{General principle}

An OPM measures the Larmor precession frequency $\omega_{\mathrm{L}}$ of a vapor sample of spin-polarized atoms in an external magnetic field $B_{0}$. In small magnetic fields

$\nu_{\mathrm{L}}=\frac{\omega_{\mathrm{L}}}{2 \pi}=\frac{\gamma_{\mathrm{A}}}{2 \pi} B_{0}$,

and the field measurement reduces to a frequency measurement. In Eq. (1) the subscript of $\gamma_{\mathrm{A}}$ refers to the total angular momentum of the precessing atomic state. Although OPMs based on nuclear-spin polarization were demonstrated in the past $[1,3,5,6]$, we restrict the present discussion to alkali vapors in which the precessing levels are one or both of the hyperfine ground states with total angular momentum $F=I \pm 1 / 2$, where $I$ is the nuclear spin. Magnetic resonance is used to measure the precession frequency by inducing resonant spin flips by a weak magnetic field $B_{1}$ oriented at right angles with respect to $B_{0}$ and oscillating at the frequency $\omega_{\mathrm{rf}}$. Although for the magnetometer discussed here $\omega_{\mathrm{rf}}$ lies in the audio range of frequencies, the index ' $r f$ ' (radio frequency) is used to comply with common notation.

Optical pumping with a resonant circularly polarized light beam creates spin polarization in the medium (roomtemperature alkali atom vapor contained in a glass cell) and hence an associated net bulk magnetization. It has been realized for many years that the pumping process is most efficient for $D_{1}$ resonance light driving the transition $\left|n S_{1 / 2}\right\rangle \rightarrow\left|n P_{1 / 2}\right\rangle$, although magnetometers can also be realized using $D_{2}\left(\left|n S_{1 / 2}\right\rangle \rightarrow\left|n P_{3 / 2}\right\rangle\right)$ light. In general, the optically pumped medium becomes transparent with respect to the pumping light, except for the spectrally resolved closed $\left|n S_{1 / 2} F\right\rangle \rightarrow\left|n P_{3 / 2}, F+1\right\rangle$ transition [7], in which case the absorption of the pumped medium increases. The fact that the optical properties of the medium depend on its spin polarization is used to detect the magnetic resonance transition by monitoring either the power or the polarization of the transmitted or scattered light beam. The technique is known as optically detected magnetic resonance (ODMR).

In the present study we have used a particular realization of the ODMR technique, the so-called $M_{x}$ method, in which $B_{0}$ is oriented at $45^{\circ}$ with respect to the direction of propaga- tion $(\widehat{k})$ of the circularly polarized light beam. The particular feature of that technique is that the transmitted light intensity is modulated at the frequency $\omega_{\mathrm{rf}}$ of the oscillating field, when $\omega_{\mathrm{rf}}$ is tuned close to $\omega_{\mathrm{L}}$. The amplitude of the modulation depends on $\sin 2 \theta$ with $\cos \theta=\widehat{B_{0}} \widehat{k}$. The amplitude and phase of the modulation depend on $\omega_{\mathrm{rf}}$ as a classical Lorentz oscillator with a resonance frequency $\omega_{\mathrm{L}}$. On resonance, the phase shift between the oscillating $B_{1}$ field and the transmitted light modulation is $90^{\circ}$ and, for a small detuning $\delta \omega=\omega_{\mathrm{rf}}-\omega_{\mathrm{L}}$, the phase shift varies linearly with $\delta \omega$. The width of the resonance(s) are determined by the transverse relaxation rate of the spin polarization, which is limited by several effects. In atom-atom collisions only the sum of the angular momenta of the collision partners is preserved but spin-exchange processes can change the individual polarizations. The rate of spin-exchange depolarization is proportional to the collision rate, i.e. to the vapor density, the spin-exchange cross section, and the relative velocities of the collision partners. The dominant depolarization mechanism is due to collisions of the atoms with the cell walls and the depolarization rate depends on the adsorption time of the atoms on the walls and on the wall collision rate. This process can be suppressed by either preventing the atoms from reaching the walls through the addition of an inert buffer gas or by reducing the sticking time on the walls by a suitable coating of the wall surfaces by paraffin or silanes. However, these coatings may act as a sink for alkali atoms, thereby significantly lowering the atomic density [8]. A stable vapor pressure is established by having the vapor in thermal equilibrium with a droplet of alkali metal contained in a side arm. Depolarizing collisions with the bulk metal are suppressed by connecting the cell proper to the side arm via a small aperture.

The intrinsic line width resulting from the combined action of the mentioned depolarization effects depends on temperature, quality of the wall coating, and cell geometry. Besides those intrinsic broadening mechanisms, the interactions with the optical field and with the oscillating magnetic field further broaden the magnetic resonance line. These processes are known as optical and rf power broadening, respectively.

\section{2}

\section{Effects of hyperfine structure}

The ground state of alkali atoms with a nuclear spin $I$ splits into two hyperfine levels with total angular momenta $F_{ \pm}=I \pm 1 / 2$ with $2 F_{ \pm}+1$ Zeeman sublevels labeled by the magnetic quantum number $M$, respectively. The general evolution of the hyperfine levels in a magnetic field is described by the Breit-Rabi formula [9]. In low magnetic fields (Zeeman interaction « hyperfine interaction) the energy of the state $\left|F_{ \pm}, M\right\rangle$ is shifted by $\Delta E_{ \pm, M}=g_{ \pm} \mu_{\mathrm{B}} B_{0} M$. Here, $\mu_{\mathrm{B}}$ is the Bohr magneton, and the $g$-factors $g_{ \pm}$are given by

$g_{+}=+\frac{1}{2 I+1} g_{J}-\frac{2 I}{2 I+1} g_{I}$,
$g_{-}=-\frac{1}{2 I+1} g_{J}-\frac{2 I+2}{2 I+1} g_{I}$,

where $g_{J}>0$ is the electronic $g$-factor, defined via $\mu_{J}=$ $-g_{J} \mu_{\mathrm{B}} J / \hbar$, and $g_{I}$ is the nuclear $g$-factor, defined via 
$\vec{\mu}_{I}=g_{I} \mu_{\mathrm{B}} I / \hbar$. The magnetic resonance process consists in driving transitions between adjacent sublevels with a resonance frequency $\omega_{\mathrm{L}}$ given by

$v_{\mathrm{L}}=\left|\frac{\Delta E_{ \pm, M+1}-\Delta E_{ \pm, M}}{h}\right|=\left|\frac{g_{ \pm} \mu_{\mathrm{B}} B_{0}}{h}\right|$,

which is equivalent to Eq. (1) with

$\gamma_{\mathrm{F}}=g_{ \pm} \mu_{\mathrm{B}} / \hbar$.

For ${ }^{133} \mathrm{Cs}$ one has

$\gamma_{4} / 2 \pi=+3.4986 \mathrm{~Hz} / \mathrm{nT}$,

$\gamma_{3} / 2 \pi=-3.5098 \mathrm{~Hz} / \mathrm{nT}$.

To second order in the field $B_{0}$, the levels acquire an additional energy shift depending on $M^{2}$ and $B_{0}^{2}$ (quadratic Zeeman effect), which shifts the $\left|F_{ \pm}, M\right\rangle \rightarrow\left|F_{ \pm}, M+1\right\rangle$ transition frequency by an additional amount

$\left|\Delta v_{\mathrm{L}}^{(2)}\right|=\left(\frac{\left(g_{J}+g_{I}\right) \mu_{\mathrm{B}} B_{0}}{h(2 I+1)}\right)^{2} \frac{2 M+1}{v_{\mathrm{hfs}}}=\frac{\epsilon}{2} B_{0}^{2}$,

where $\nu_{\mathrm{hfs}}$ is the ground-state hyperfine splitting. The quadratic Zeeman effect thus splits the magnetic resonance into a series of equidistant lines separated by

$\delta v^{(2)}=\epsilon B_{0}^{2}$.

For cesium $(I=7 / 2)$, one has

$\epsilon=2.6716 \mathrm{nHz} / \mathrm{nT}^{2}$.

In the $2-\mu \mathrm{T}$ field used here $\delta v^{(2)}=0.011 \mathrm{~Hz}$, which is much smaller than the resonance line width. The low-field approximation (Eq. (1)) is therefore valid for the present work.

\subsection{Practical realization}

In this work we compare the performance of lamppumped and laser-pumped magnetometers. Both devices have a common basic design consisting of the light source, the sensor head, the detector, and feedback electronics (Figs. 1 and 2). The sensor head contains the sensor proper, a spherical glass cell $(60-\mathrm{mm}$ diameter for the LpOPM, produced in the group of one of the authors, ASP, and 70-mm diameter for the LsOPM, purchased from MAGTECH Ltd., St. Petersburg,

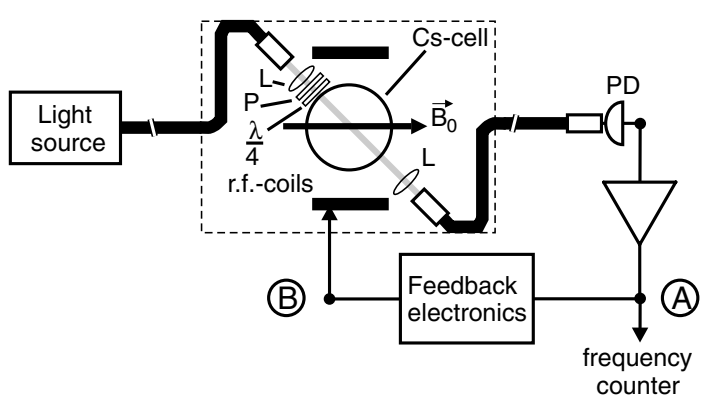

FIGURE 1 Principle of the $M_{x}$ magnetometer. The light source is the discharge lamp or the diode laser as explained in the text. $L:$ lens, $P$ : polarizer and $D_{1}$ interference filter (in case of lamp pumping), $\lambda / 4$ : quarter-wave plate, $P D$ : photodiode a)

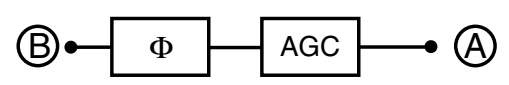

b)

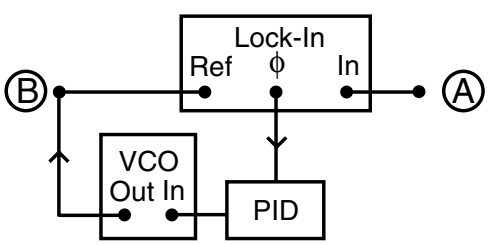

FIGURE 2 Scheme of feedback electronics in the self-oscillating mode (a) and in the phase-stabilized mode (b). AGC: amplitude gain control, $\Phi$ : phase shifter, $V C O$ : voltage-controlled oscillator, $P I D$ : feedback controller

Russia) coated with paraffin in which cesium vapor is in thermal equilibrium with a droplet of metallic cesium at room temperature. The cell is mounted in a 200 -mm-long, 110mm-diameter cylindrical housing. The pumping light is carried from the light source to the sensor cell by a multimode fiber ( $800-\mu \mathrm{m}$ diameter) in the LsOPM and by a fiber bundle (6-mm diameter) in the LpOPM. The light transmitted through the cell is carried back to a detector (photodiode) by an identical fiber in the LsOPM and by an 8-mm-diameter fiber bundle in the LpOPM. The lengths of the fibers are 8 and $5 \mathrm{~m}$, respectively, for the laser- and the lamp-pumped devices. The sensor head also contains polarization optics (linear polarizer and quarter-wave plate) for making the light circularly polarized prior to entering the cell, as well as lenses for collimating the incoming light and focussing the outgoing light into the return fiber (bundle). Particular care was taken to use only non-magnetic components in the sensor head. The coils producing the oscillating field consist of two 70-mm-diameter loops with 12 turns of copper wire each, separated by $52 \mathrm{~mm}$. When two sensors are operated in close proximity, the crosstalk of the respective off fields is avoided by sliding a 1-mmthick Al cylinder over the heads. The optical, electronic, and mechanical components of the LsOPM were produced at the University of Fribourg, while the LpOPM was realized at the Ioffe Institute.

The characterization of the magnetometers described in Sect. 3.1 was performed in Fribourg. Two magnetometers were placed inside a multilayer cylindrical magnetic shield as shown in Fig. 3 and Table 1. The magnetic field of $2 \mu \mathrm{T}$ was produced by a $50-\mathrm{cm}$-long, $15-\mathrm{cm}$-diameter solenoid driven by an ultra-low-noise current supply.

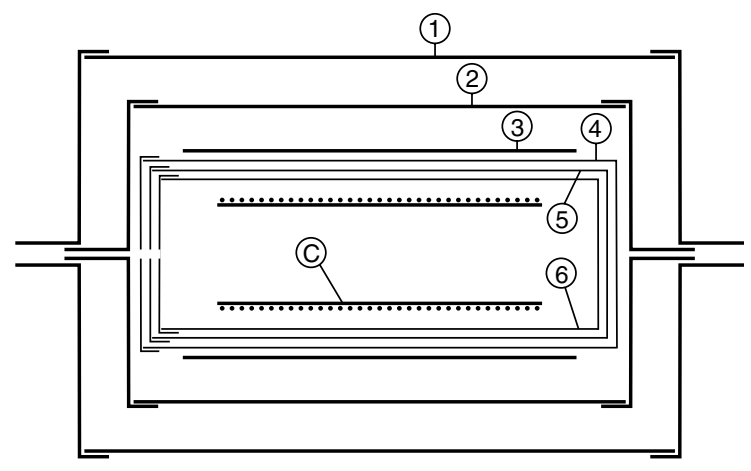

FIGURE 3 Scheme of the Fribourg shield. The labels 1-6 denote the different layers as described in Table 1. $C$ : magnetic field coil 


\begin{tabular}{lllll}
\hline Layer & $d(\mathrm{~mm})$ & $l(\mathrm{~mm})$ & $t(\mathrm{~mm})$ & Material \\
\hline 1 & 600 & 900 & 1.5 & Mumetal \\
2 & 450 & 750 & 1.5 & Mumetal \\
3 & 300 & 600 & 1.5 & Mumetal \\
4 & 285 & 743 & 0.76 & Co-Netic \\
5 & 256 & 714 & 0.76 & Co-Netic \\
6 & 229 & 686 & 0.76 & Co-Netic \\
\hline
\end{tabular}

TABLE 1 The Fribourg magnetic shield. $d$ : inner diameter, $l$ : inner length, $t$ : layer thickness. Note that all layers are closed by end caps except for layer 3

For a direct comparison of the noise performance with an accuracy below $0.1 \mathrm{pT}$, the level of magnetic field fluctuations at the experimental site has to be kept below that level, a performance which is hard to realize. Magnetic field variations (in a $1-\mathrm{Hz}$ bandwidth) in unshielded environments are of the order of several $\mathrm{nT}_{\mathrm{rms}}$ or more. A shielding factor exceeding 10000 is thus needed to suppress fluctuations at that level.

\subsection{Features of the LsOPM and the LPOPM}

In both types of magnetometers the $D_{1}$ transition $\left|6 S_{1 / 2}\right\rangle \rightarrow\left|6 P_{1 / 2}\right\rangle$ of $\mathrm{Cs}$ at a wavelength of $894 \mathrm{~nm}$ is used for optical pumping. The LpOPM is driven by an electrodeless discharge lamp, in which a power-stabilized high-frequency generator $(\sim 100 \mathrm{MHz})$ produces a discharge in a 12-mmdiameter glass bulb containing cesium vapor and xenon as buffer gas. The pumping light is collimated and filtered by a $D_{1}$ interference filter centered at $894.5 \mathrm{~nm}$ with a FWHM = $11.5 \mathrm{~nm}$. Because of the high temperature of the discharge plasma, the spectrum of the emitted $D_{1}$ radiation is considerably broader than the Doppler width of the room-temperature absorption line in the sensor cell. All four hyperfine components of the $D_{1}$ line are excited simultaneously as indicated in Fig. 4a. As the same light is used for detecting the groundstate spin precession, the LpOPM detects magnetic resonance in both the $F=4$ and the $F=3$ hyperfine ground states. Because of the differing $g$-factors of the two states (Eq. (5)), the corresponding magnetic resonance lines are split by $22 \mathrm{~Hz}$ in the $2-\mu T$ field, which is larger than the width of the magnetic resonance lines $(\sim 2.5-5 \mathrm{~Hz})$ under optimized conditions. The $F=3$ component is much weaker than the $F=4$ component, so that the former plays a minor role for magnetometry.

The LsOPM is pumped by a tunable extended cavity laser in Littman configuration (Sacher Lasertechnik, model

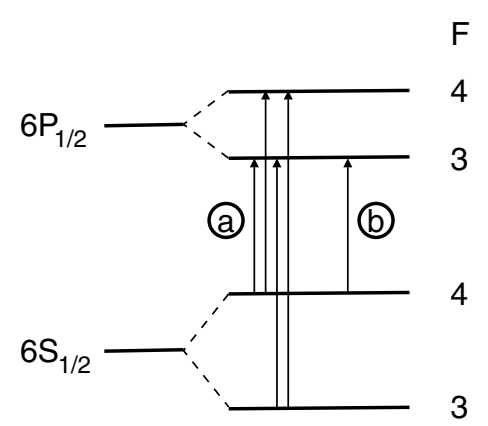

FIGURE 4 Hyperfine structure of the cesium $D_{1}$ line. The arrows indicate the transitions driven by the discharge lamp (a) and the laser (b)
TEC500). The output power of more than $10 \mathrm{~mW}$ exceeds the power required for magnetometry by more than three orders of magnitude. Therefore, a single laser can be used to drive dozens of magnetometers in experiments calling for the simultaneous monitoring of the magnetic field in different locations. The laser frequency is actively stabilized to the Doppler-broadened $F=4 \rightarrow F=3$ hyperfine transition using a dichroic atomic vapor laser lock (DAVLL) [10] in an auxiliary (vacuum) cesium cell. This lock is very stable and allows a reliable operation of the magnetometer over measurement periods of several weeks.

In both magnetometers the states $\left|6 S_{1 / 2} ; F=4, M=4\right\rangle$ are dark states ${ }^{1}$, which cannot absorb circularly polarized light. At the same time the repopulation of absorbing (bright) states by the magnetic resonance rf transitions from these levels is used for the optical detection of the magnetic resonance. The LsOPM drives the $\left|6 S_{1 / 2} ; F=4\right\rangle \rightarrow \mid 6 P_{1 / 2} ; F=$ $3\rangle$ transition, and excited atoms can decay into any of the sublevels of the $\left|6 S_{1 / 2} ; F=3\right\rangle$ hyperfine state, which do not interact with the narrow-band light. This process, called hyperfine pumping, degrades the efficiency of the optical pumping process and reduces the overall spin polarization that can be achieved with laser radiation. A straightforward way to reduce that loss is to empty the $\left|6 S_{1 / 2} ; F=3, M\right\rangle$ sublevels using a repumping laser tuned to a transition emanating from the $F=3$ hyperfine ground state. An experimental study of that process is under way. In the LpOPM all four hyperfine components are pumped simultaneously. As a result, the loss due to hyperfine pumping is excluded and a larger spin polarization is obtained in the $F=4$ state. The drawback of the large spectral width of the beam from the lamp is that an appreciable part of its spectrum lies outside the room-temperature absorption spectrum of the sensor cell. The corresponding photons carry no spectroscopic information, but produce excess shot noise in the detected photocurrent. In that respect, narrow-band laser light leads to a better detection efficiency in the LsOPM.

\subsection{Modes of operation}

Both types of magnetometers were operated in two different modes. The self-oscillating mode (SOM) (Fig. 2a) uses the fact that at resonance the driving rf field and the modulated photocurrent are dephased by $90^{\circ}$. For that reason the sinusoidal part of the photocurrent can be used, with an appropriate amplification and phase shift to drive the rf coils in a feedback loop. In such a configuration the system will auto-oscillate at the Larmor frequency. An amplitude gain control ensures that the amplitude of the coil current is kept constant independently of phase and frequency, so that any rf power dependent systematic effects are suppressed.

The phase-stabilized mode (PSM) (Fig. 2b) also uses the characteristic phase dependence between the applied oscillating field and the modulation of the detected photocurrent for locking the frequency of an external oscillator to the Larmor frequency. The phase, the in-phase component, and the quadrature component are detected simultaneously by a

${ }^{1}$ Owing to the resolved hyperfine structure in the LsOPM, the states $\left|6 S_{1 / 2} ; F=4, M=3\right\rangle$ and $\left|6 S_{1 / 2} ; F=3, M\right\rangle$ are also dark states. 
lock-in amplifier (Stanford Research Systems SR830). Both the in-phase signal (dispersive Lorentzian) and the $90^{\circ}$ phaseshifted phase signal (arctan dependence) show a linear zero crossing near zero detuning $\left(\omega_{\mathrm{rf}}=\omega_{\mathrm{L}}\right)$. Either of the two signals can thus be used as discriminant in a feedback loop, which stabilizes the phase to $90^{\circ}$. It can be shown that from a statistical point of view both signals yield an equivalent magnetic field sensitivity. The phase signal is less sensitive to light power fluctuations, which may be advantageous to suppress systematic effects related to power fluctuations [11]. However, the commercial digital lock-in amplifier used had only a moderate update rate of $400 \mathrm{~Hz}$ of its phase output, so that the much faster in-phase signal was used in the feedback loop.

In principle the PSM can be understood as a variant of the SOM, in which the phase detector, the voltage-controlled oscillator (VCO), and the feedback controller form a tracking filter. In both modes of operation changes of the magnetic field lead to instantaneous changes of the Larmor frequency and thus to instantaneous changes of the transmitted modulation frequency. The time needed for the radio frequency to adjust to a new value after a sudden field change depends on the filters and other delays in the feedback loop. If a very fast response is not required, as in our applications, the bandwidth can be decreased by appropriate filters. In the SOM it is the preamplifier of the photodetector which limits the bandwidth to $10 \mathrm{kHz}$, whereas in the PSM the feedback-loop filters provide a bandwidth up to $1 \mathrm{kHz}$ [4]. From a practical point of view, the registered bandwidth is limited by the dataacquisition system. Because of the frequency dependence of the phase shifter the SOM is, in general, optimized only for a given Larmor frequency, which reduces the dynamic range of the SOM device. The phase-stabilized magnetometer keeps the phase shift at $90^{\circ}$ independently of the Larmor frequency. In practice, the dynamic range is limited by the frequency range of the voltage-controlled oscillator used to generate the oscillating field. The long-term stability of both feedback schemes is limited by temperature-dependent phase drifts.

The Al shield slipped over each magnetometer for avoiding cross-talk effectively acts as a low-pass filter for external magnetic field fluctuations (skin effect), which reduces the response bandwidth of all devices to about $250 \mathrm{~Hz}$ (Fig. 5).

\section{3}

\section{Performance}

3.1

\section{Magnetometric sensitivity: basics and fundamental limit}

The sensitivity of the magnetometer is defined as the noise equivalent magnetic flux density (NEM), which is the flux-density change $\delta B$ equivalent to the total noise of the detector signal. In a perfectly stable external magnetic field the smallest detectable field changes are limited by the intrinsic magnetometer noise $\delta B_{\text {int }}$. For a measurement bandwidth $\Delta v_{\text {bw }}$ the intrinsic resolution $\delta B_{\text {int }}$ depends on the magnetic resonance line width $\Delta v$ (HWHM) and on the signal-to-noise ratio $S / N_{\text {int }}$ of the magnetometer signal according to

$\delta B_{\text {int }}=\frac{1}{\gamma} \frac{\Delta v}{S / N_{\text {int }}}$.

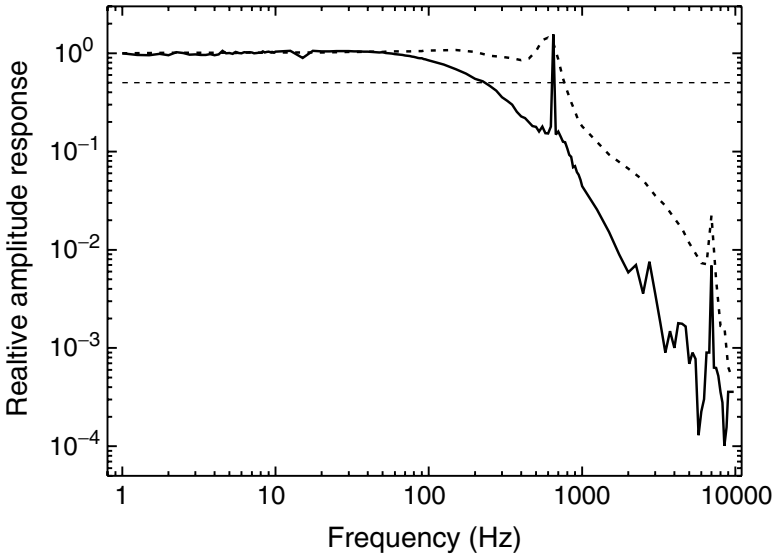

FIGURE 5 Normalized amplitude response of the LsOPM (PSM) to a periodic magnetic field change, measured at the VCO input: with Al shield (full line) and without $\mathrm{Al}$ shield (dashed line). The horizontal dashed line indicates an amplitude response of 0.5 . In both cases the loop filter was adjusted to be about $750 \mathrm{~Hz}$. By using the $\mathrm{Al}$ shield the bandwidth is reduced to about $250 \mathrm{~Hz}$. Note that using the phase-stabilized LpOPM yields the same result, since the bandwidth is independent of the light source

For a feedback-operated magnetometer using an optically thin medium $(\kappa L \ll 1)$ the light power (expressed in terms of photocurrent) detected after the sensor cell is given by

$I_{\mathrm{pc}}=I_{\mathrm{in}} \exp (-\kappa L) \approx I_{\mathrm{in}}(1-\kappa L)$,

where $I_{\text {in }}$ is the incident power, $\kappa$ the resonant optical absorption coefficient, and $L$ the sample length. The absorption coefficient can be written as

$\kappa=\kappa_{0}\left(1+\eta \cos \omega_{\mathrm{L}} t\right)$,

where $\kappa_{0}$ is the mean absorption coefficient and $\eta$ the modulation depth, which depends - among other things - on the degree of spin polarization and the amplitude of the oscillating field. We can then write Eq. (10) as

$I_{\mathrm{pc}}=I_{0}+I_{\mathrm{m}} \cos \omega_{\mathrm{L}} t=I_{0}\left(1+\xi \cos \omega_{\mathrm{L}} t\right)$,

where $I_{0}=I_{\text {in }}\left(1-\kappa_{0} L\right)$ and $I_{\mathrm{m}}=\eta \kappa_{0} L I_{\text {in }}=\xi I_{0}$. The contrast $\xi$ is the ratio of the modulation amplitude and the average photocurrent in the approximation $\kappa_{0} L \ll 1$. The signal $S$ is given by the rms value of the oscillating part of the magnetometer signal

$S=\xi I_{0} / \sqrt{2}$.

The fundamental limit of the magnetometric sensitivity is obtained for a shot-noise-limited signal, with a noise level $N_{\text {int }}=N_{\mathrm{SN}}=\sqrt{2 e I_{0} \Delta \nu_{\mathrm{bw}}}$ of $I_{\mathrm{pc}}$. The shot-noise-limited sensitivity then reads

$\delta B_{\mathrm{SN}}=\frac{\Delta v}{\gamma} \frac{2}{\xi} \sqrt{\frac{e \Delta v_{\mathrm{bw}}}{I_{0}}}$.

The magnetometer signal of interest is contained in the amplitude and phase of the sinusoidal modulation of the light power after the sensor cell. The power spectrum of the spectral power density of an ideal magnetometer in a perfectly stable magnetic field thus consists of a delta function centered at the Larmor frequency, superposed on a flat background of shot-noise fluctuations. In practice, the peak is broadened by 
the resolution $(1 \mathrm{~Hz})$ of the fast Fourier transform analyzer (Stanford Research Systems, model SR760) used for its recording. The relevant noise contributions which define the $S / N$ ratio are fluctuations of the photocurrent at the Larmor frequency, i.e. the value of the background below the Larmor peak. In practice, that ideal spectrum is modified by various imperfections which degrade the magnetometer performance. In the following, we address contributions from light power fluctuations and magnetic field fluctuations.

\subsection{Limitations by light power fluctuations}

The light power $I_{\text {in }}$ has a continuous (technical) noise spectrum, which lies above the shot-noise level, in particular at frequencies below $100 \mathrm{~Hz}$, as shown for the laser in Fig. 6. The individual peaks are even and odd harmonics of the $50-\mathrm{Hz}$ line frequency. Power fluctuations contribute to the photocurrent noise at $\omega_{\mathrm{L}}$ by two distinct processes. First, there is a direct contribution via the noise component of $I_{0}$ in Eq. (12) at the Fourier frequency $\omega_{\mathrm{L}}$. At $\omega_{\mathrm{L}} / 2 \pi=7 \mathrm{kHz}$ this noise level is close to the shot-noise level. The second contribution is due to the second term in Eq. (12). Each Fourier component (at $\omega$ ) of the power fluctuations is multiplied by $\cos \omega_{\mathrm{L}} t$, and this mixing produces sidebands at $\omega_{\mathrm{L}} \pm \omega$ in the power density spectrum. In this way the continuous lowfrequency part of the technical noise around $\omega=0$ (Fig. 6) produces a symmetric background under the Larmor peak. Although the power noise around $\omega=0$ is 18 times (Fig. 6) larger than the shot noise around $\omega=\omega_{\mathrm{L}}$, it is suppressed - according to Eq. (12) - by a factor $\xi$, which has a value of approximately 0.05 in the LsOPM. As a consequence, the contribution of the modulation term in Eq. (12) to the photocurrent noise is less than the contribution from $I_{0}$. We have verified that this is indeed fulfilled in a carefully calibrated auxiliary experiment.

\subsection{Limitations by magnetic field fluctuations}

In the presence of uncorrelated magnetic field fluctuations, $\delta B_{\text {ext }}$, the highest resolution with which a magnetic

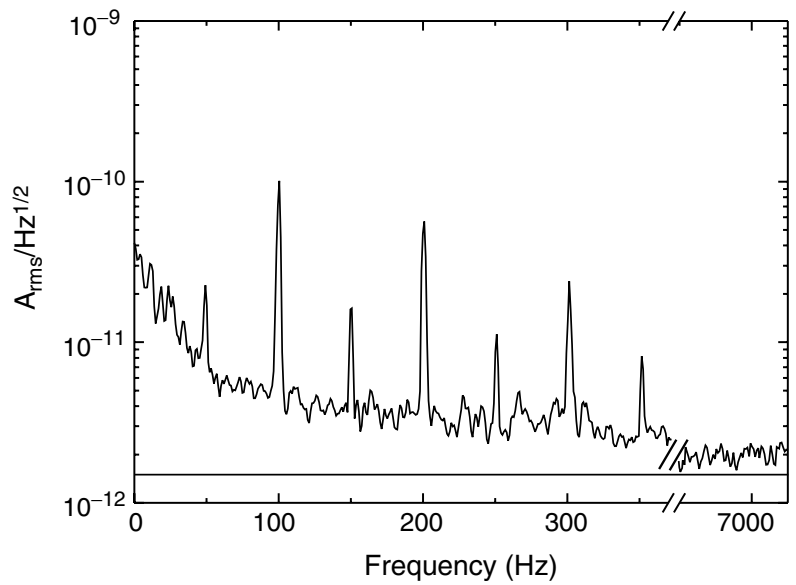

FIGURE 6 Power spectral density of the low-frequency noise of the laser power. The power was $13 \mu \mathrm{W}$ and the corresponding shot-noise level is shown as a solid line. The noise at $7 \mathrm{kHz}$, which lies $50 \%$ above the shot-noise level, is also shown field change can be detected is given by $\delta B=\sqrt{\delta B_{\text {int }}^{2}+\delta B_{\text {ext }}^{2}}$. The fluctuations $\delta B_{\text {ext }}$ of the external magnetic field can be parameterized by the equivalent noise $N_{\text {ext }}$ that they produce on the signal, and $\delta B$ can be expressed in a form similar to Eq. (9) by

$\delta B=\frac{1}{\gamma} \frac{\Delta v}{S / N}$

where $N^{2}=N_{\mathrm{int}}^{2}+N_{\mathrm{ext}}^{2}$. Fourier components of the field fluctuations at frequency $\omega$ will mix with the magnetometer oscillation frequency $\omega_{\mathrm{L}}$ in Eq. (12). Monochromatic field fluctuations, such as the $50-\mathrm{Hz}$ line frequency and harmonics thereof, produce symmetric sidebands, while low-frequency magnetic field fluctuations produce a continuous background underlying the Larmor peak.

\subsection{Measurement of the intrinsic sensitivity}

The intrinsic line width of the magnetic resonance transition was measured by extrapolating the experimental line width to zero light power and zero rf power. We found a HWHM of $1.63 \mathrm{~Hz}$ for the cell in the LpOPM and $2.35 \mathrm{~Hz}$ for the cell in the LsOPM. As all cells were manufactured by the same person, the difference of the intrinsic line widths is

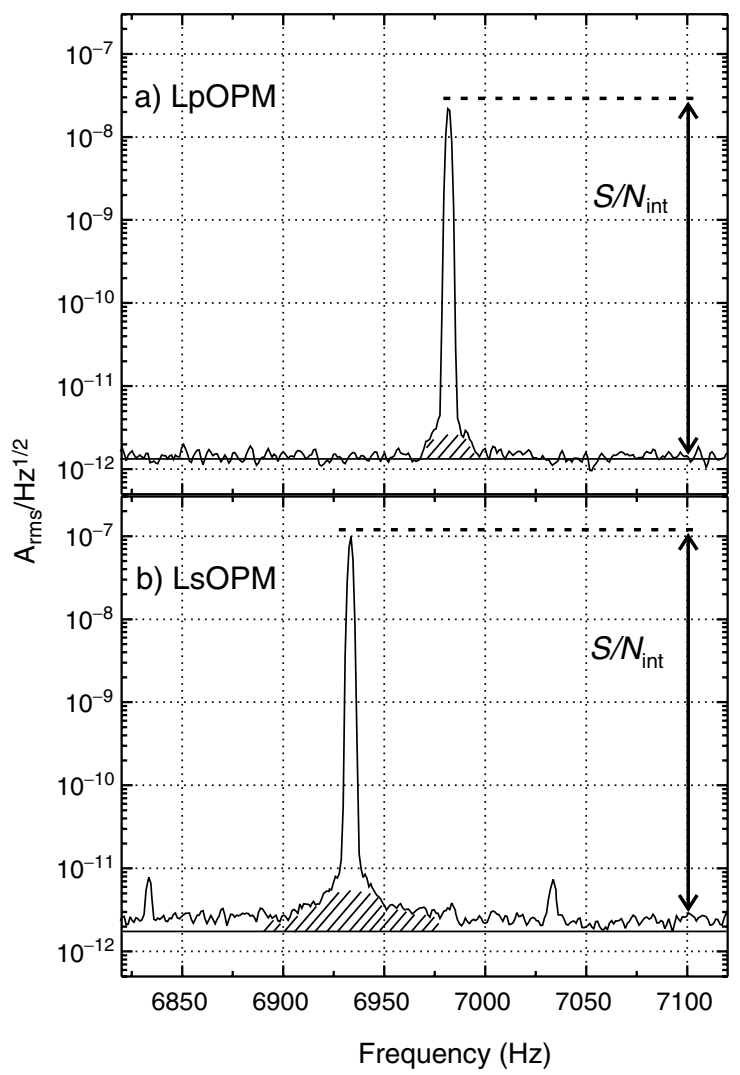

FIGURE 7 Power spectral density plots of the photocurrent fluctuations in the LpOPM (a) and the LsOPM (b), both operated in the phase-stabilized mode. The signal-to-noise ratios $S / N_{\text {int }}$ are 29000 (a) and 98000 (b), respectively. The photocurrents are $4.3 \mu \mathrm{A}$ for the LpOPM and $5 \mu \mathrm{A}$ for the LsOPM. The corresponding shot-noise levels are represented by the horizontal lines. The dashed areas indicate the pedestal discussed in the text 


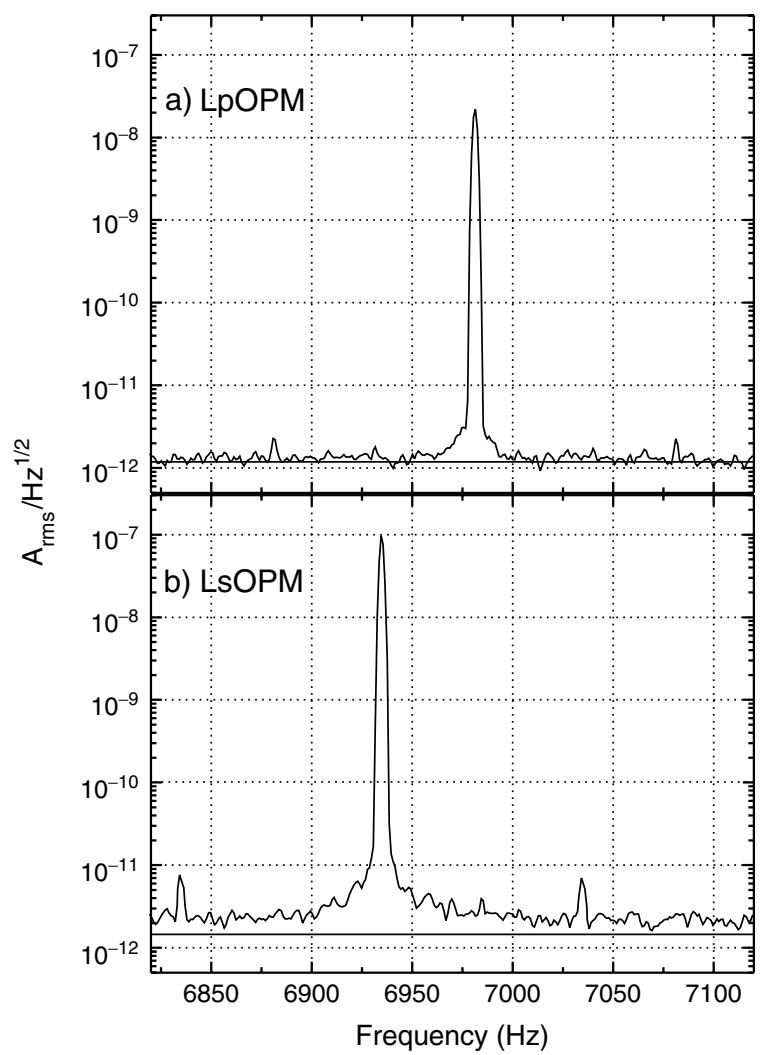

FIGURE 8 Power spectral density plots of the photocurrent fluctuations in the LpOPM (a) and the LsOPM (b), both operated in the self-oscillating mode. The signal-to-noise ratios are similar to those in Fig. 7 and the corresponding shot-noise levels are represented by horizontal lines

probably due to the slightly larger aperture between the cell and the side arm in the case of the LsOPM. After optimizing the signal-to-noise ratio with respect to light and rf power the (power-broadened) magnetic resonance line width (HWHM) is $\Delta v=2.5 \mathrm{~Hz}$ for the LpOPM and $5.0 \mathrm{~Hz}$ for the LsOPM.

Figures 7 and 8 show typical power density spectra recorded in the phase-stabilized and self-oscillating modes of operation - of the rms voltage fluctuations at the output of the current-to-voltage preamplifier of the photodiode current. The spectra contain all structures discussed above. The pedestal (indicated as a dashed area in Fig. 7) underlying the Larmor peak contains contributions from fluctuations of the light power and of the magnetic field. The white-noise floor in the far wings of the central structure represents the intrinsic OPM noise $N_{\text {int }}$. Its numerical value for use in Eq. (9) was measured $70 \mathrm{~Hz}$ above the carrier. At that frequency this noise represents the noise component of $I_{0}$ of the power noise in Eq. (12). We have verified that the noise component of the modulated contribution in Eq. (12) is eight times less than the noise contribution of $I_{0}$ under the present experimental conditions. The pedestal under the Larmor peak is thus most probably due to low-frequency field fluctuations.

After optimizing the magnetometric sensitivity with respect to light power and rf power, the LpOPM yields a $S / N$ ratio of 29000 , while the LsOPM reaches 98000 in a bandwidth $\Delta v_{\mathrm{bw}}$ of $1 \mathrm{~Hz}$. It can be seen from the figures that the signal-to-noise ratio does not depend on the mode of operation (SOM or PSM). For the LpOPM the shot-noise level is nearly reached while in the LsOPM $N_{\text {int }}=1.5 \times N_{\mathrm{SN}}$. According to Eq. (9) the measured $S / N$ ratios and line widths under optimized conditions result in a NEM $\delta B_{\text {int }}$ of $25 \mathrm{fT}$ for the LpOPM and of $15 \mathrm{fT}$ for the LsOPM in a bandwidth of $1 \mathrm{~Hz}$. The LsOPM is thus 1.7 times more sensitive than the LpOPM, although its performance is not yet shot-noise limited.

\subsection{Discussion}

In order to get a better understanding of the excess power noise in the LsOPM we measured the dependence of the photocurrent noise on the light power for the laser in comparison to that for the lamp. In those measurements only the noise component of $I_{0}$ (Eq. (12)) at $7 \mathrm{kHz}$ was recorded as it represents the dominant noise contribution of $I_{\mathrm{pc}}$. For each of the measurements the cesium cell was removed and the light beam was detected directly by the photodiode using a transimpedance amplifier for the photocurrent. The noise $N_{0}$ measured in this way gives a lower limit of the intrinsic magnetometer performance. The results for the laser and lamp sources are shown in Fig. 9. The noise can be written as

$N_{0}^{2}=N_{\mathrm{SN}}^{2}+N_{\text {dark }}^{2}+N_{\mathrm{T}}^{2}$,

where $N_{\mathrm{SN}}$ is the shot noise of the photocurrent, $N_{\text {dark }}$ the intrinsic detector (photodiode and amplifier) noise, and $N_{\mathrm{T}}$

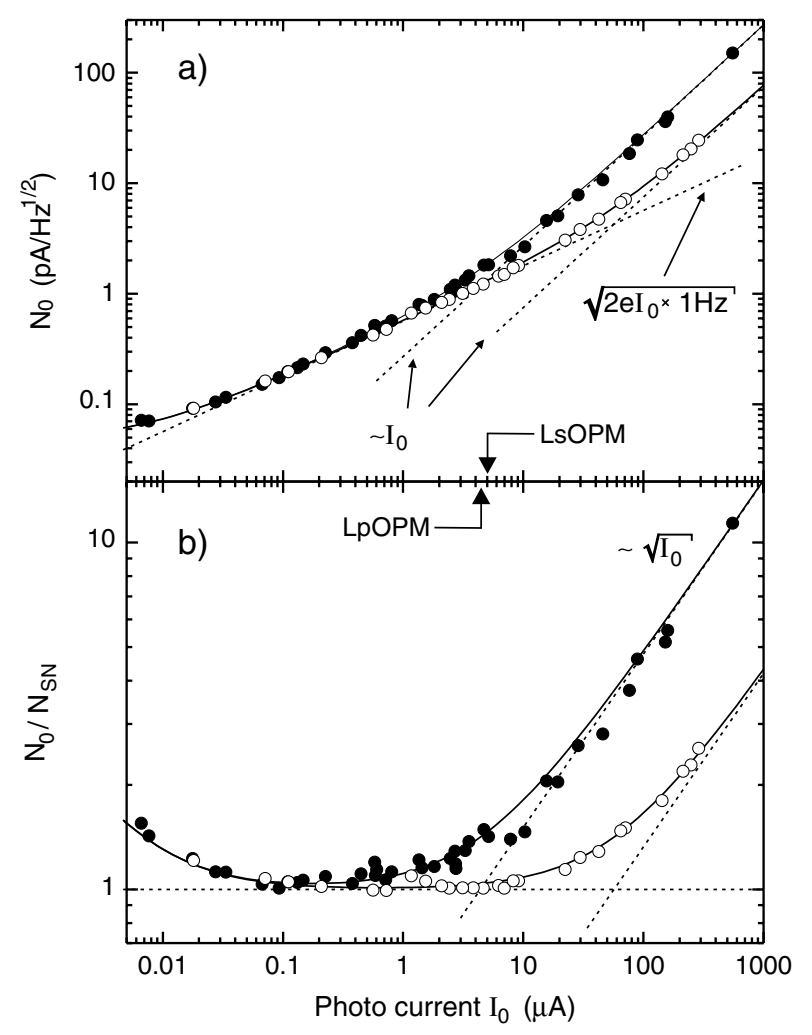

FIGURE 9 a Measured noise $N_{0}$ of the photocurrent produced by laser light (black dots) and by light from the discharge lamp (open circles) at $7 \mathrm{kHz}$. The measurement bandwidth was $1 \mathrm{~Hz}$. The dashed lines indicate the calculated photodiode shot noise $\left(\propto \sqrt{I_{0}}\right)$ and the fitted technical laser noise $\left(\propto I_{0}\right)$. b Ratio of $N_{0}$ to the calculated shot noise $N_{\mathrm{SN}}$. The technical noise appears to be proportional to $\sqrt{I_{0}}$ in this representation. The full lines in both plots represent the calculated sum of all noise contributions according to Eq. (16). The arrows refer to the (optimized) photocurrent for both devices 
the technical noise of the light source, which is proportional to the photocurrent $I_{0}$.

$N_{\text {dark }}$ was measured with the light beams blocked for all (discrete) amplification stages of the transimpedance amplifier. With the highest amplification $\left(10^{-1} \mu \mathrm{A} / \mathrm{V}\right)$ used for $I_{0}<600 \mathrm{nA}$ we measured $N_{\text {dark }}=48 \mathrm{fA} / \sqrt{\mathrm{Hz}}$.

This corresponds to the shot noise of a current $I_{0}$ of $7 \mathrm{nA}$, so that above $7 \mathrm{nA}$ the intrinsic detector noise can be neglected. This dark current is responsible for the deviation of the measured noise from the (dotted) shot-noise line (Fig. 9) at low currents.

For larger photocurrents, the technical noise $N_{\mathrm{T}}$, which is proportional to the light power, dominates over the shot noise. We found $N_{\mathrm{T}}=k I_{0}$, with $k_{\mathrm{Ls}}=2.6 \times 10^{-7}$ and $k_{\mathrm{Lp}}=$ $0.8 \times 10^{-7}$ for the laser and the lamp sources, respectively. With the laser source the shot noise $N_{\mathrm{SN}}$ becomes equal to the technical noise $N_{\mathrm{T}}$ for a photocurrent $I_{0}$ of $4.9 \mu \mathrm{A}$, which thus yields a noise level $N_{0}=\sqrt{2} N_{\mathrm{SN}}$ at that photocurrent. The laser power for optimized magnetometer parameters corresponds to $5 \mu \mathrm{A}$, thereby explaining the excess noise of the LsOPM in Figs. 7 and 8. In the case of lamp pumping the technical noise becomes important for $I_{0}>60 \mu \mathrm{A}$, so that the magnetometer is shot-noise limited for the (optimized) photocurrent of $4.3 \mu \mathrm{A}$.

If one succeeds in eliminating the excess noise of the laser power, e.g. by an active power stabilization of the LsOPM, one can achieve an intrinsic shot-noise-limited sensitivity of $10 \mathrm{fT}$, thereby outperforming the LpOPM by a factor of 2.5 . This is compatible with earlier results [12] obtained from a comparative study of lamp-and laser-pumped magnetometers using ${ }^{39} \mathrm{~K}$, in which the sensitivity of the LsOPM version was found to be 2.3 times higher than of the corresponding LpOPM device. It is also interesting to compare those results with the present results on an absolute scale. The sensitivity of the ${ }^{39} \mathrm{~K}$ LsOPM was found to be $1.8 \mathrm{fT} / \sqrt{\mathrm{Hz}}$. This superior performance compared to the ${ }^{133} \mathrm{Cs}$ magnetometer discussed here is mainly due to the two times larger diameter of the potassium sensor cell, which, combined with the appreciably smaller spin-exchange cross section of potassium, led to an operating line width of $1 \mathrm{~Hz}$ compared to $5 \mathrm{~Hz}$ with the present Cs LsOPM. Furthermore, the $g$-factor of ${ }^{39} \mathrm{~K}$ is twice as large than that of ${ }^{133} \mathrm{Cs}$. These two factors explain the superior performance of the $\mathrm{K}$ magnetometer ( $1.8 \mathrm{fT}$ ) compared to the Cs magnetometer (15 fT).

\section{4}

4.1

\section{Applications}

\section{Direct comparison of LSOPM and LPOPM}

We performed a direct comparison of the performance of the LpOPM and the LsOPM in simultaneous measurements of magnetic field fluctuations inside the multilayer shield described above (Fig. 3). Both devices were mounted coaxially in the shield and the centers of their sensor cells were separated by $21 \mathrm{~cm}$. The oscillatory signal of each magnetometer was filtered by a resonance amplifier, centered near $7 \mathrm{kHz}$ with a FWHM of $500 \mathrm{~Hz}$, and analyzed by a frequency counter (Stanford Research Systems, model SR620) with a gate time of $0.1 \mathrm{~s}$. An example of such a recording over a con-

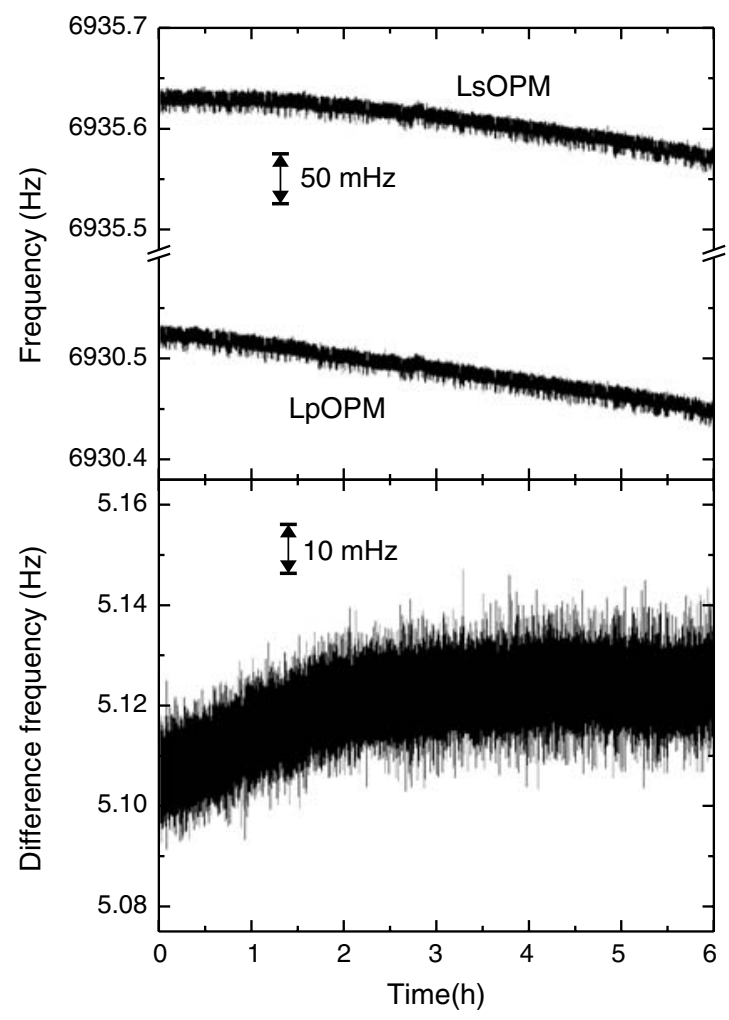

FIGURE 10 Fluctuations of the Larmor frequency recorded with the LpOPM (PSM) and the LsOPM (SOM) recorded over $6 \mathrm{~h}$ (top traces). The difference of both frequencies shows a drift of the field gradient (bottom trace)

tinuous interval of $6 \mathrm{~h}$ with the LpOPM operated in PSM and the LsOPM operated in SOM is shown in Fig. 10. One sees that both devices oscillate at different average frequencies, which can be explained by the presence of a magnetic field gradient of $66 \mathrm{pT} / \mathrm{cm}$ that drifts by $0.4 \%$ over the $6-\mathrm{h}$ interval. The drift is probably due to a thermal drift of the shield's magnetization. Similar gradients were measured after interchanging the positions of the two OPMs. Figure 11 shows a 5-min time slice of the data in Fig. 10. There are highly correlated irregular field jumps of approximately $3.6 \mathrm{pT}$ in both traces. These fluctuations correspond to relative fluctuations of the solenoid current at a level of $10^{-6}$ and are suspected to be caused by the current source. The Allan standard deviation [13] of the data is a convenient way for characterizing the field drifts on various time scales. Figure 12 shows the Allan plot of the data from Fig. 10 as a function of integration time both in absolute and in relative units. Both magnetometers show the same field stability and the data points are indistinguishable for small integration times. While the short-term stability is governed by white noise, the bump between 1 and $200 \mathrm{~s}$ is due to the irregular field jumps. The long-term stability is determined by long-term field drifts of the imperfectly shielded external field and thermal drifts of the solenoid support structure.

\subsection{Systematic effects}

The magnetometric performance is affected by the following systematic effects. Any deviations of the phase shift $\delta \phi$ from the optimum value of $-\pi / 2$ in the loop will change 


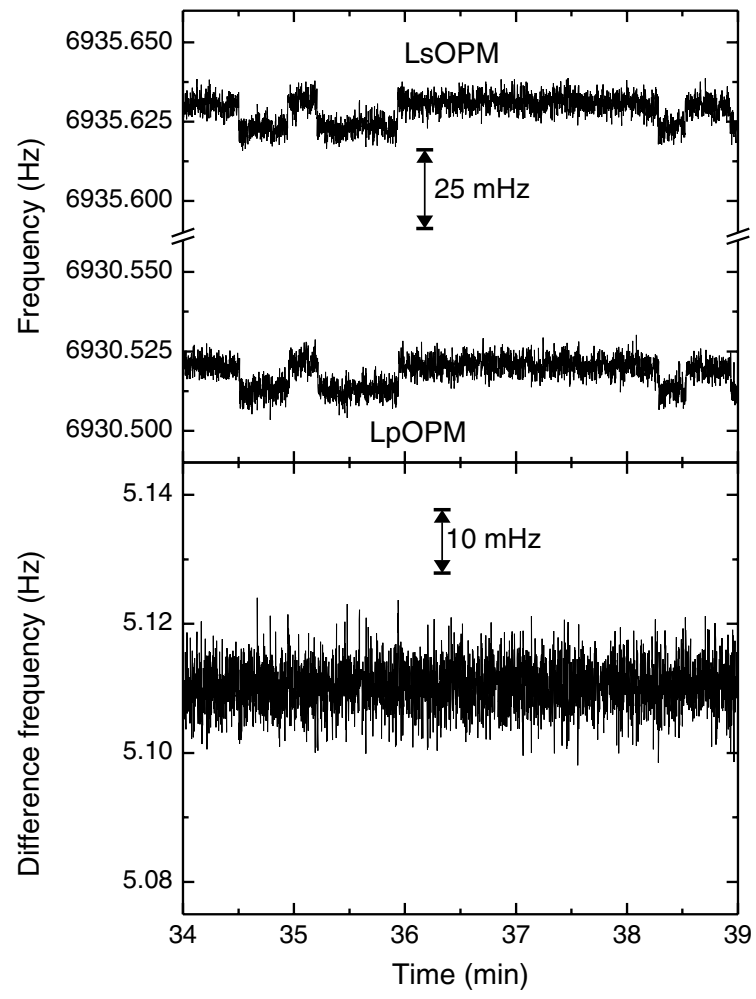

FIGURE 11 Five-minute slices of the traces in Fig. 10. Individual readings of the magnetometers (top) and of their difference frequency (bottom)

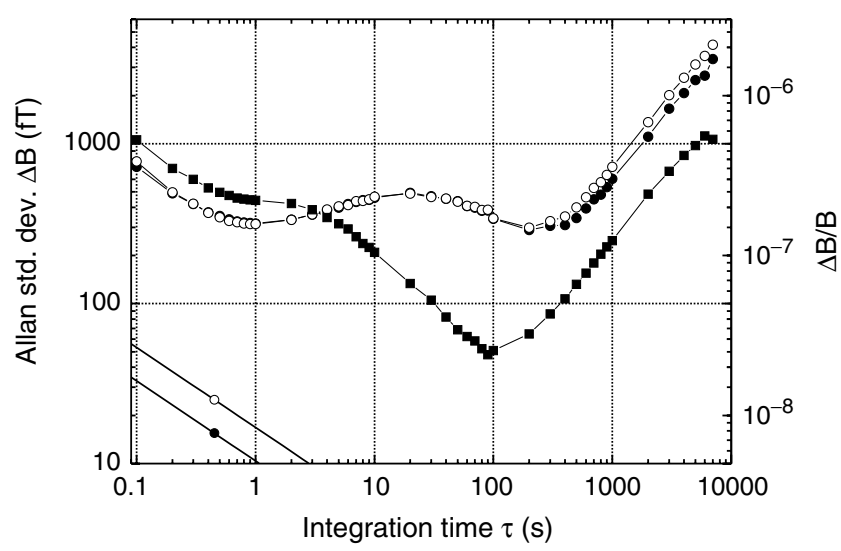

FIGURE 12 Allan standard deviation of the time traces in Fig. 10. Open circles: LpOPM measurement, black dots: LsOPM measurement, black squares: field gradient measured over $21 \mathrm{~cm}$. For times below $200 \mathrm{~s}$ the LsOPM data points and the LpOPM data points overlap and cannot be distinguished. The lines are drawn to guide the eye. In the two lower graphs the single points at $\tau=0.5 \mathrm{~s}$ indicate the NEM of the LpOPM (open circle) and of the LsOPM (black dot), and their extrapolation to other integration times assuming white noise

the measured frequency by an amount $\delta v=\Delta \delta \phi$. As $\delta \phi$ cannot be controlled on a very high level this type of magnetometer is not a highly accurate device. However, as long as the phase shift is kept constant, e.g. by temperature stabilizing the feedback electronics, the sensitivity is not affected.

When the laser frequency is detuned from resonance the Larmor frequency will be systematically changed by the light shift, which acts as an effective additional magnetic field. In that situation light power fluctuations will also limit the sensitivity. Light shift effects are less dramatic in the case of lamp pumping as the broad spectrum of the resonance lines from the lamp cancels the dispersively shaped light shift dependence. At the level of field fluctuations reported here, false effects induced by the light shift are negligible. Details of this are discussed in [4].

As shown in Sect. 2.2 the different Zeeman transitions cannot be resolved in a magnetic field of $2 \mu \mathrm{T}$. Only in magnetic fields between 10 and $200 \mu \mathrm{T}$, where the quadratic Zeeman splitting is of the order of the resonance line width, does the resonance line appear to be asymmetric and light power fluctuations induce fluctuations of that asymmetry, which then yield frequency changes.

\subsection{Active field stabilization}

We have further investigated the performance of the magnetometers in an active magnetic field stabilization system using a phase-locked loop. For that purpose the phase of the LpOPM oscillation (PSM) relative to the phase of a reference oscillator was measured by a lock-in amplifier and used as error signal driving a correction coil. While field fluctuations common to both sensors are strongly suppressed by this method, gradient drifts and fluctuations are not compensated and thus detected by the free-running magnetometer. The performance of the stabilization scheme is shown in Fig. 13,
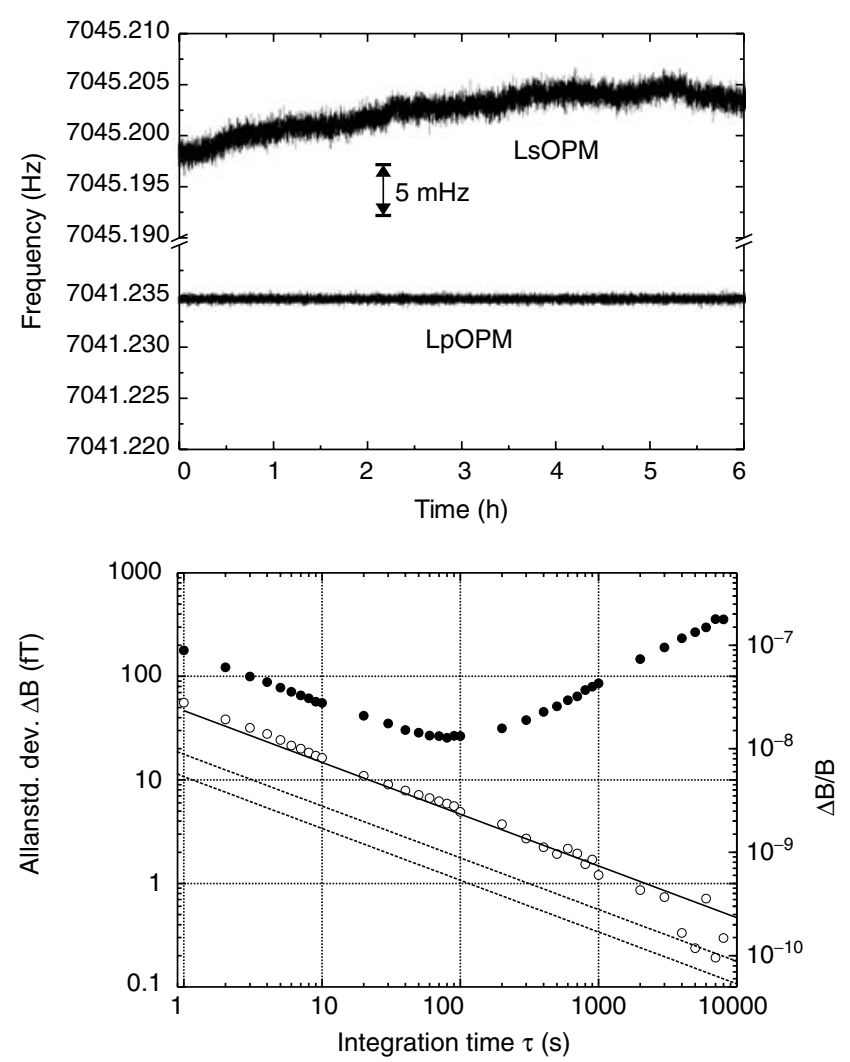

FIGURE 13 Field fluctuations in both sensors when the field is stabilized to the LpOPM signal. Upper graph: time series of the residual fluctuations, recorded with a 1-s gate time of the frequency counter. Lower graph: Allan standard deviations of the time traces: LpOPM (open circles) and LsOPM (black dots). The solid line represents the resolution limit of the frequency counter. The dashed lines indicate the NEM of the LpOPM (upper) and of the LsOPM (lower) 
where the top traces represent time series of the field readings (1-s gate time of the frequency counter) of the free-running LsOPM and the LpOPM used for the feedback. The lower part of Fig. 13 shows the Allan standard deviation of those data. The jumps in the magnetic field measured by the LsOPM (Fig. 11) are completely suppressed by the feedback loop and only white noise in the short-term stability and contributions from gradient drifts in the long-term stability remain. The field fluctuations reach a minimum of $25 \mathrm{fT}$ for $\tau=100 \mathrm{~s}$, which corresponds to a relative field stability of $1.3 \times 10^{-8}$. Due to the fact that the field is stabilized to the LpOPM, one would expect a significant lowering of the LpOPM's Allan standard deviation for short integration times. The white-noise behavior (slope $-1 / 2$ in the Allan plot) of the LpOPM trace is entirely due to the resolution of the frequency counter, which is limited by trigger time jitter due to amplitude noise of the measured sine wave.

We also realized a setup in which the roles of the LsOPM and the LpOPM were reversed. The observed performance was identical with the one described.

\section{$5 \quad$ Further applications}

OPMs based on Cs vapor are well suited for operation in magnetic fields smaller than $10 \mu \mathrm{T}$ (such as typical fields used in neutron EDM experiments). As already mentioned above, in such fields asymmetries of the magnetic resonance line due to the quadratic Zeeman effect will not affect their long-term stability (accuracy). In magnetic fields above $300 \mu \mathrm{T}$ the quadratic Zeeman shift exceeds the line width and the magnetic resonance spectrum consists of eight resolved lines [14]. This offers a further possibility for the use of cesium magnetometers. The performance of the LsOPM in that field range is expected not to be worse than the one measured in a $2-\mu \mathrm{T}$ field. This expectation is based on the fact that the (performance-determining) laser power noise does not increase with frequency, so that a shot-noise-limited sensitivity can be reached. A magnetometer based on the quadratic Zeeman effect in potassium has been demonstrated earlier [15].

In contrast to buffer-gas cells, which require the whole cell volume to be illuminated in order to achieve a maximum sensitivity, paraffin-coated cells can be pumped with a laser beam of much smaller diameter. This allows one to adapt the spatial dimensions of the paraffin-coated sensors to specific experimental requirements. One can think, for exam- ple, of using very large cells of several liters for measuring volume-averaged fields. Compared to other proposed largecell schemes [3], the use of a Cs OPM offers the further advantage of a high temporal resolution. Recently, a novel type of optically pumped magnetometer with a sub-fT (gradiometric) sensitivity was demonstrated [16]. Besides its use of very high buffer gas pressures and its operation at a temperature of $190^{\circ} \mathrm{C}$, specific features of that magnetometer are its limited operation range near zero field and its reduced bandwidth of $20 \mathrm{~Hz}$.

ACKNOWLEDGEMENTS We thank G. Bison for fruitful discussions and careful reading of this manuscript. We acknowledge financial support from Schweizerischer Nationalfonds, INTAS, and Paul Scherrer Institute (PSI). One of the authors (ASP) acknowledges financial support from the University of Fribourg.

\section{REFERENCES}

1 K. Green, P.G. Harris, P. Iaydjiev, D.J.R. May, J.M. Pendlebury, K.F. Smith, M. van der Grinten, P. Geltenbort, S. Ivanov, Nuvl. Instr. and Meth. A 404, 381 (1998)

2 I.S. Altarev, Y.V. Borisov, N.V. Borovikova, A.I. Egorov, S.N. Ivanov, E.A. Kolomensky, M.S. Lasakov, V.M. Lobashev, V.A. Nazarenko, A.N. Pirozhkov, A.P. Serebrov, Y.V. Sobolev, E.V. Shulgina, Phys. At. Nuclei 59, 1152 (1996)

3 Y. Borisov, W. Heil, M. Leduc, V. Lobashev, E. Otten, Y. Sobolev, Nucl. Instrum. Methods A 440, 48 (2000)

4 S. Groeger, J.-L. Schenker, R. Wynands, A. Weis, Phys. Rev. A, submitted [arXiv:physics/0406105 v2 19 August 2004]

5 O. Moreau, B. Cheron, H. Gilles, J. Hamel, E. Noel, J. Phys. III 7, 99 (1997)

6 H. Gilles, J. Hamel, B. Cheron, Rev. Sci. Instrum. 72, 2253 (2001)

7 A.P. Kazantsev, V.S. Smirnow, A.M. Tumaikin, I.A. Yagofarov, Opt. Spectrosc. 57, 189 (1984)

8 E.B. Alexandrov, M.V. Balabas, D. Budker, D. English, D.F. Kimball, C.-H. Li, V.V. Yashchuk, Phys. Rev. A 66, 042903 (2002)

9 G. Breit, I.I. Rabi, Phys. Rev, 38, 2082 (1931)

10 V.V. Yashchuk, D. Budker, J.R. Davis, Rev. Sci. Instrum. 71, 341 (2000)

11 G. Bison, R. Wynands, A. Weis, J. Opt. Soc. Am. B 22, 77 (2005)

12 E.B. Aleksandrov, M.V. Balabas, A.K. Vershovskii, A.E. Ivanov, N.N. Yakobson, V.L. Velichanskii, N.V. Senkov, Opt. Spectrosc. 78, 325 (1995)

13 J.A. Barnes, A.R. Chi, L.S. Cutler, D.J. Healey, D.B. Leeson, T.E. McGunigal, J.A. Mullen, Jr., W.L. Smith, R.L. Sydnor, R.F.C. Vessot, G.M.R. Winkler, IEEE Trans. Instrum. Meas. 20, 105 (1971)

14 D. Nettels, R. Müller-Siebert, S. Ulzega, A. Weis, Appl. Phys. B 77, 563 (2003)

15 E.B. Alexandrov, A.S. Pazgalev, J.L. Rasson, Opt. Spectrosc. 82, 14 (1997)

16 I.K. Kominis, T.W. Kornack, J.C. Allred, M.V. Romalis, Nature 422, 596 (2003) 